\title{
Gynecologic Cytopathology
}

\section{Organized Cervical Cancer Screening Program in Barretos, Brazil: Experience in 18 Municipalities of São Paulo State}

\author{
Júlio César Possati-Resende ${ }^{\mathrm{a}}$ Fabiana de Lima Vazquez ${ }^{\mathrm{b}, \mathrm{c}}$ \\ Sinval Tadeu Biot ${ }^{a}$ Edmundo Carvalho Mauad ${ }^{a}$ Thais Talarico ${ }^{c}$ \\ José Humberto Tavares Guerreiro Fregnani ${ }^{b}$ Adhemar Longatto-Filho ${ }^{c-f}$ \\ ${ }^{a}$ Cancer Prevention Department, ${ }^{\mathrm{b}}$ Teaching and Research Institute, and ${ }^{\mathrm{c}}$ Molecular Oncology Center, Barretos \\ Cancer Hospital, Barretos, and ' Laboratory of Medical Investigation (LIM-14), School of Medicine, University of \\ São Paulo, São Paulo, Brazil; ' Life and Health Sciences Research Institute (ICVS), School of Health Sciences, \\ University of Minho, Braga, and 'fICVS/3B's - PT Government Associate Laboratory, Braga/Guimarães, Portugal
}

\section{Keywords}

Cervical cancer screening · Cervical cytology · Cervix ·

Cytology $\cdot$ Cervical screening programs

\begin{abstract}
Objectives: The aim of this report is to demonstrate the Barretos Cancer Hospital initiative of organizational, laboratorial, and human resources training in the implementation of an organized cervical screening program in low-resource settings. Methods: We developed a computational program to report all epidemiological, clinical, and laboratorial findings, and to trace all necessary information to recruit women for regular screening or for referral for complementary exams after liquid-based Pap test analyses. Results: All Pap tests were collected in liquid medium and in 2014 more than 160,000 tests were analyzed and 2,900 colposcopy examinations were performed. From 2012 to 2015, the percentage of exams collected increased from $54.6 \%$ in 2012 to $62.4 \%$ in $2013,68.4 \%$ in 2014 , and $71 \%$ in 2015 . Per 1,000 Pap tests, 0.4 cases of invasive cancer were diagnosed; for in situ carcinoma, 1.9 cases were identified. More importantly, between
\end{abstract}

\section{KARGER}

(C) 2017 S. Karger AG, Basel
2011 and 2015, 89.4\% of all carcinomas were detected at clinical stage 0 or I (in situ carcinoma), and only $5 \%$ at stages III and IV. Conclusions: Since the organized system was implemented, $98 \%$ of women have attended their recall for colposcopy. So far, we have not reached the target of $70 \%$ of women for this proposal, as recommended by the international standards.

(c) 2017 S. Karger AG, Basel

\section{Introduction}

Cervical cancer remains an important public health problem in developing countries due to the high incidence and mortality rates. This malignancy is the second most common cancer among women worldwide, accounting for more than 520,000 new cases and the deaths of approximately 270,000 women annually [1]. Estimates made by the Brazilian National Cancer Institute (INCA) indicate an incidence of 16,340 new cases of cervical cancer only for the year of 2016 in Brazilian women [2]. Except for nonmelanoma skin tumors, cervical cancer is more 
common among women in Northern Brazil; it is the second most frequent malignancy in the Northeast and Midwest, and is still the fourth and fifth most common form of cancer in the Southeast and South, respectively [3].

Screening programs for cervical cancer, based on the performance of Pap tests, were responsible for a dramatic reduction in the burden of this cancer in developed countries in the last few decades [4]. Basically, these results are due to the early detection strategies grounded in organized programs with high coverage rates, systematic calling and recalling of women, and efficiency in the monitoring and treatment of patients with precursor lesions that had a positive impact in reducing morbidity and mortality rates for cervical cancer, mainly in Europe, North America, Japan, Australia, and New Zealand [5].

Despite the various initiatives adopted since the early 1970s by the Brazilian authorities, such as the implementation of the National Cancer Department at the Ministry of Health $(\mathrm{MOH})$, the development of the National Program of Cancer Control, and several other programs, the mortality rates for this cancer have changed little [6]. These actions culminated in the prioritizing of the Year Control of Cervical Cancer in 2014 with the intention of reverting the dismal results that had been obtained until then.

The official data from the Ministry of Health, which can quite often be distorted due to underreporting and mistakes in survey data, indicated a mortality rate adjusted for age of 4.9 per 100,000 women, and 6 per 100,000 women for the Northern region, which has the highest rates in Brazil [7]. These numbers indicate the existence of a close association between cervical cancer and a low socioeconomic status. These striking regional differences in Brazil are due in part to the existence of numerous barriers related to access to the health services for the detection and treatment of cervical cancer and its precursor lesions. Additionally, there are barriers relating to geographical difficulties in accessing the localities where medical assistance is available, and relevant cultural issues such as fear and shame. The reversal of this historical overview is strongly associated to the consolidation of public strategies for screening cervical precursor lesions with an emphasis on increasing the coverage of the target population to the minimum level of $80-85 \%$, as recommended by the World Health Organization [8]. Concurrently, the prevention actions should be based on ensuring access to diagnosis and treatment with continuous control of the quality of services provided by the Unified Health System, which is the public health care system in Brazil.
In this regard, several studies have shown that systems driven by primary health care (PHC) have better results in the provision of care focused on strategies with greater equity and efficiency, adding to this the fact that primary attention should establish itself as the foundation of the health system capable of organizing it in its totality [9]. In Brazil, PHC is made up of a complex network responsible for the promotion and protection of health - especially in the areas of disease prevention - diagnosis, treatment, rehabilitation, and maintenance of health in the collective and individual dimensions through participative management and health interventions.

The Brazilian National Program for Control of Cervical Cancer is included in this PHC network, which controls the primary prevention actions and early detection of cervical cancer guided by the Brazilian Guidelines for Cervical Cancer Screening [6]. According to these guidelines, the Pap smear is a screening method for cervical cancer and should be offered primarily to women aged 25-64 years who have had sexual activity. Among the challenges listed by these strategic guidelines, the population growth tracked by the program, although desirable, faces the obstacle of a lack of reliable information concerning the current and real coverage of cytological examination. The figures currently available obtained through the Cervix Cancer Information System (SISCOLO) do not identify even the number of women screened, and only provide details of the number of tests performed at a given time. This situation prevents an accurate appreciation of the coverage rate, which is essential to the monitoring of the planned intervention strategies.

Finally, it is mandatory to draw an organized program to address the primary care actions of prevention, recognizing issues related to possible deficiencies mainly related to the structural facilities and professional human resources. The present work aims to respond to questions regarding the difficulties and limitations of implementing an organized program for cervical cancer prevention, as well as highlighting the findings that already show favorable indicators of the quality of the preliminary results.

\section{Methodology}

Study Design

This was a retrospective analytical study.

Location of the Study

This study was designed in the region of Barretos, SP, Brazil, in the cervical cancer screening program of the Prevention Institute at Barretos Cancer Hospital (BCH) during the period between 2012 and 2015.
Possati-Resende et al. 


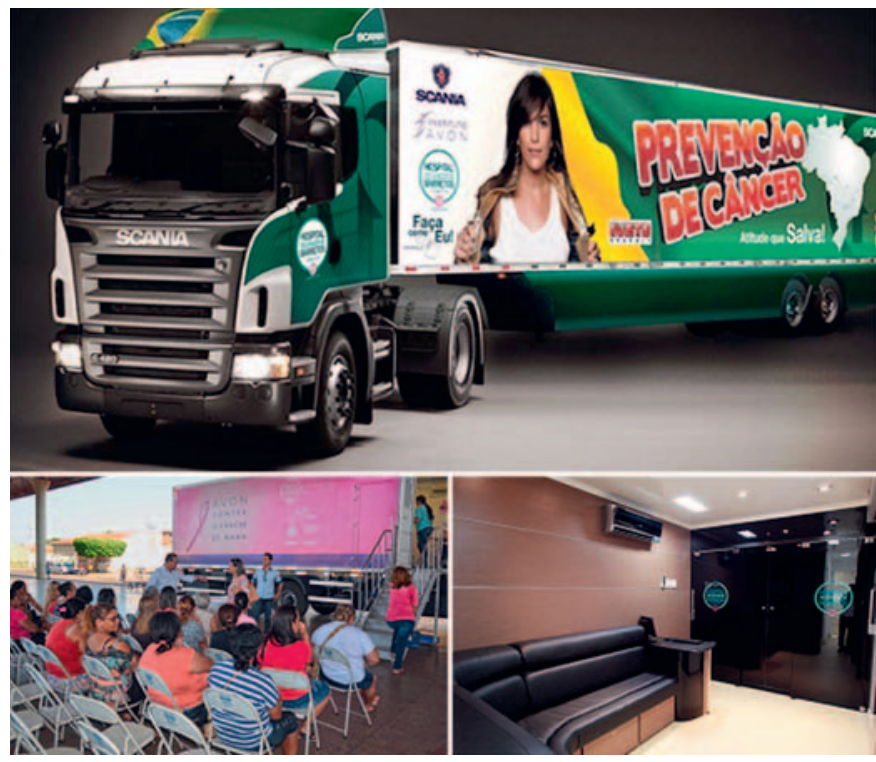

Fig. 1. Mobile unit of care for the collection of Pap smears, with details of the waiting room.

Barretos region has 18 municipalities called health regional districts (DRS-V). Women aged between 25 and 64 years are invited annually to participate in the cervical cancer prevention program through the health service units of their municipalities. To estimate the number of eligible women, data from the Brazilian Institute of Geography and Statistics (IBGE) [10] are used, based on their age and municipality, regardless of having participated in a possible opportunistic screening or having had a hysterectomy. The work was approved by the institute's committee on human research and thus meets the standards of the Declaration of Helsinki in its revised version of 1975 and its amendments of 1983, 1989, and 1996.

\section{Collection of Cervical Cytology: Pap Test}

The Pap smear was performed in mobile units (Fig. 1), at the $\mathrm{PHC}$, or Prevention Institute at BCH. Most of the Pap tests were stored using liquid-based cytology (SUREPATH ${ }^{\circledR}$ ) methodology, which allowed us to standardize the preparation and staining of slides and reduce the number of unsatisfactory samples. It also allowed the possibility of making additional slides and further molecular testing, providing increased sensitivity in detecting any existing injuries, and also the improvement in reading performance of cytotechnologists and pathologists. Additionally, we opted for liquid-based cytology due to the possibility to perform a human papilloma virus (HPV) test if necessary. Figure 2 exemplifies an extremely poor-quality cervical smear versus a liquid-based cytology preparation. All sample analyses were performed in the laboratory of $\mathrm{BCH}$.

\section{Computerized System for Organized Screening}

In March 2012, the Prevention Institute of BCH implemented a computerized system developed on the Oracle platform (SISHOSP) to manage an organized cervical cancer screening program for women of the Barretos region. SISHOSP also used the database of the hospital, which already had related information. This system, designed by one of the authors (J.C.P.-R.), records sociodemographic information, the results of previously performed cervical cytology screening (Pap), adequacy of collected samples, follow-up, and additional tests, such as colposcopy, when necessary.

\section{Organized Screening: Letter of Invitation to Women}

Letters were mailed out inviting all women within the age range of 25-64 years who were already registered on SISHOSP and were not up to date with their Pap smear, as well as to women for whom a Pap smear should be repeated during the period when the mobile unit was in the municipality in which they lived. The invitations sent to women followed the screening protocol, and the exams are routinely done at intervals of 3 years after 2 annual negative controls.

\section{Management of the Organized Program}

The program is able to individually identify the date of each woman's last Pap test and automatically generate a new date on which she must repeat the exam based on the previous cytological report and the time interval between the 2 collections. Additionally, the system is able to identify women who are not up to date with their screening tests, automatically generating convocation letters. The criteria internally recognized by the computerized system that determines the Pap repeat periods and the convening of the suspected cases are based on the recommendations established by the Ministry of Health through the Brazilian Guidelines for Screening for Cervical Cancer (Rio de Janeiro, 2016).

\section{Program Interfaces}

The system interface displays all the registration information of each woman and triggers an alert for a consultation at the $\mathrm{BCH}$ by telephone or via a standardized e-mail or letter. With the implementation of the computerized system, every woman receives the final report of their cytological examination at her home address and also receives a printed guide in the actual report regarding the date of the next collection. The possibility of managing information enables us to execute and forward alerts via written correspondence for woman with guidance on examinations that need to be repeated immediately or who are scheduled according to the data from the $\mathrm{BCH}$ database. Currently, women living in the Barretos region already routinely receive these alerts at their residence. $\mathrm{Pa}$ tients with abnormal tests who require recall for further clinical assessments are automatically listed and recommended for appointment scheduling for a colposcopy exam at the Prevention Institute.

\section{Quality Control of the Tests}

Another important objective of this system is to carry out the quality control of the tests, taking into account the monitoring of both the collection of samples and the laboratory analysis. The control of the collection of cytology specimens covers the quality of the specimens collected in the health centers of the municipalities of the Barretos region, in mobile prevention units and at the Prevention Institute of BCH. For each Pap test, data are stored on the professional responsible for sample collection and the adequacy of the sample, including information on the presence or absence of endocervical cells, which is considered a criterion of quality for morphological examination. The stored data are evaluated month- 
Fig. 2. Liquid-based cytology versus conventional Pap smear.

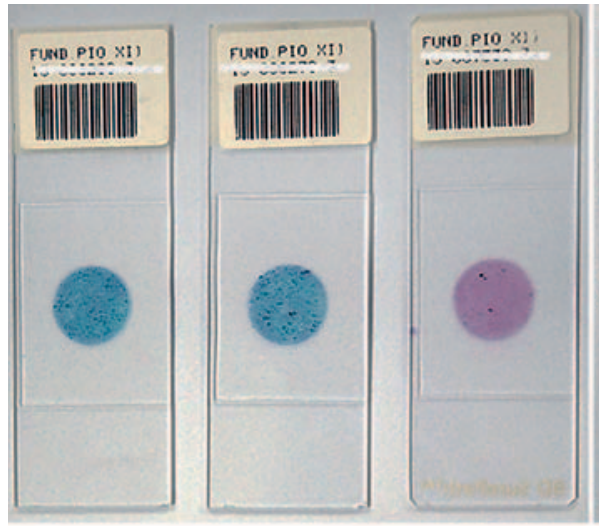

LIQUID-BASED CYTOLOGY

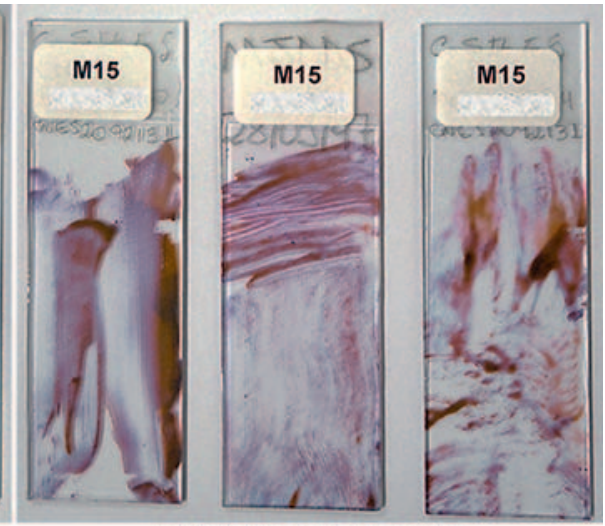

CONVENTIONAL PAP SMEAR

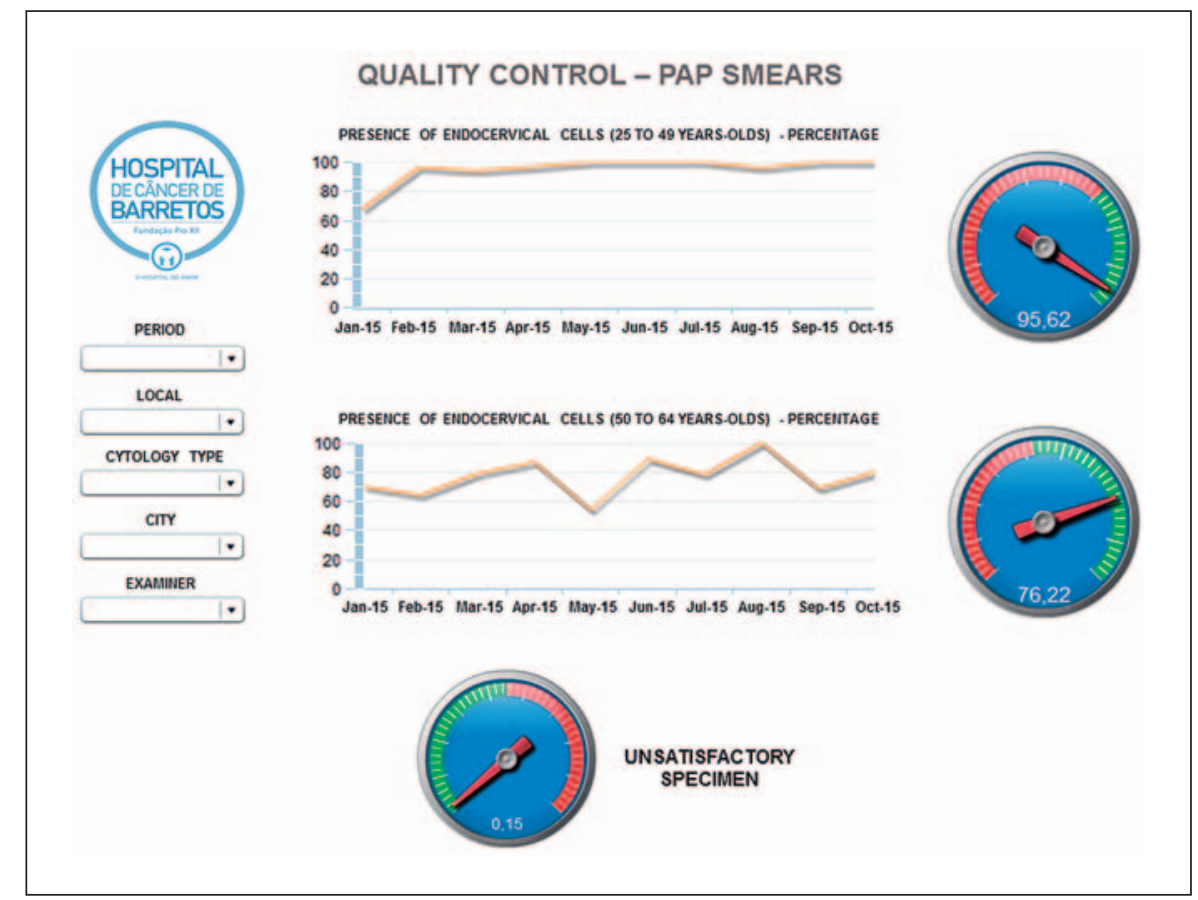

Fig. 3. Example of quality analysis of samples collected, taking into consideration the individual performance of each examiner.

ly, taking into consideration the quality of the collection in each health unit or municipality, or evaluating the performance of each examiner individually, whether nurse or medical doctor. All indicators are grouped and analyzed by a business intelligence program (SADIG ${ }^{\circledR}$, São Paulo, Brazil) that allows the construction of custom dashboards that can be quickly and easily interpreted. Figures 3 and 4 depict examples of the functionality of SADIG.

Laboratory quality indicators are assessed according to criteria established by the Ministry of Health [9], taking into account variables such as the positivity rate, percentage of precursor lesions, the atypical squamous cell (ASC) to squamous intraepithelial lesion (SIL) ratio, and percentage of diagnosis of atypical cells of undetermined significance (ASCUS). These globally accepted variables [11] are evaluated together as well as individually for each pathologist.
Monitoring Pap Tests of the Target Population

The computerization of real operational tracking allows us to evaluate the target population coverage ratios that make up the Barretos region (covering a total of 18 cities) in relation to the realization of the Pap test. This information is critically important for providing subsidies for community intervention actions. The group of women aged between 25 and 64 years in the region of Barretos (named DRS-V) totals about 109,000 subjects eligible for exams for the prevention of cervical cancer [10].

\section{Monitoring the Interval between Screening Tests and}

Complementary Exams

We are constantly trying to minimize the time intervals between test collections without experiencing a loss of quality. With this goal, the computer system performs continuous monitoring 


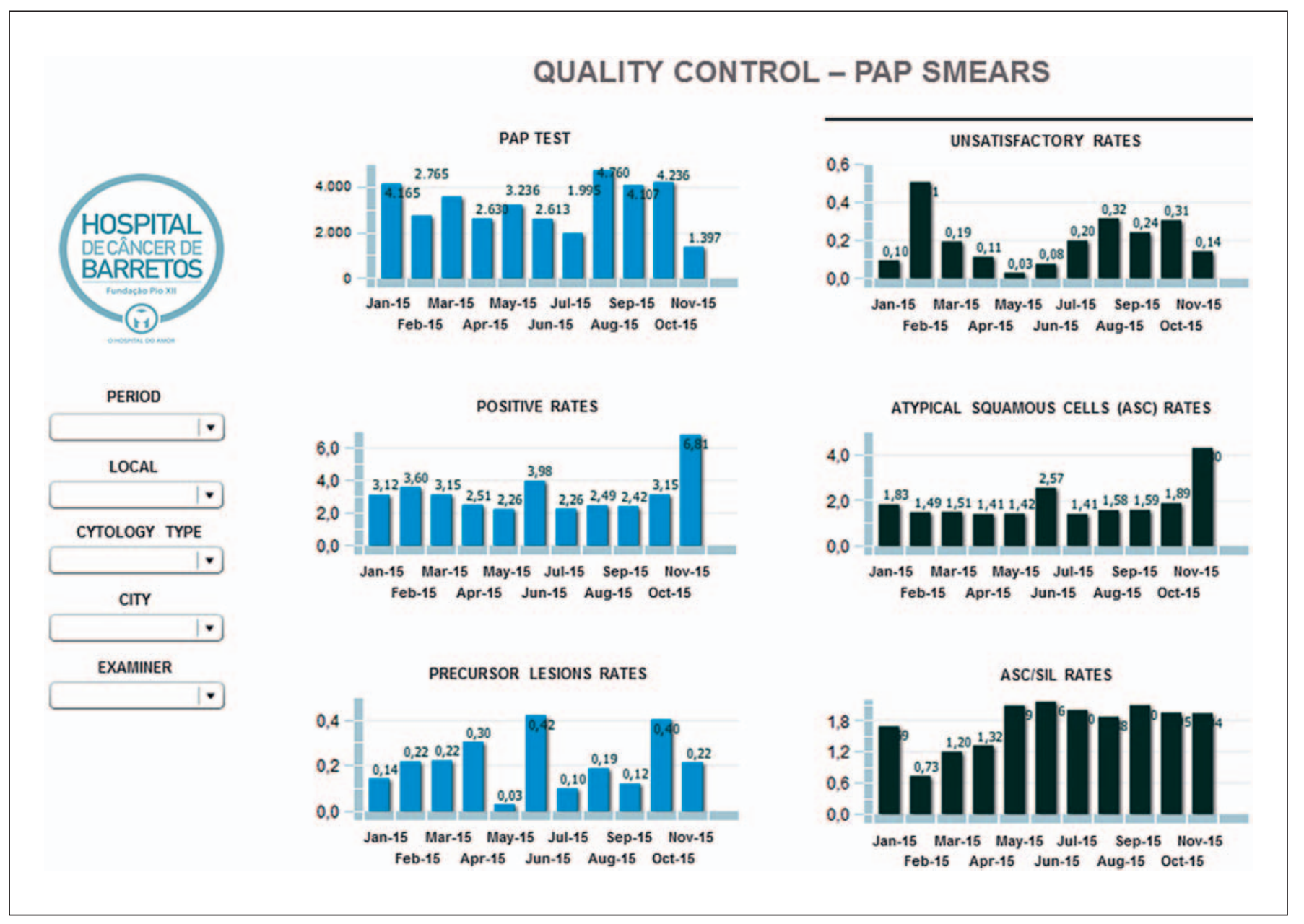

Fig. 4. Screen interface used for the evaluation of laboratory parameters of the quality of cervical cytology.

of the different stages of tracking, which allows us to identify possible inconsistencies and outline intervention strategies in relation to the release of the final report, the delivery of results to women, and call and recall for additional clinical exams for abnormal cases.

In summary, the organized screening program has a multiprofessional team covering several essential requirements such as:

1. collaboration of the health secretariats of the 18 cities that are part of DRS-V and that participate actively in the prevention program;

2. continuous training of health professionals responsible for community education (nurses, doctors, and community health workers);

3. active participation of the health professionals (community health agents) of the Basic Health Units that guide and invite women to attend the mobile units, the Basic Health Units, or the Prevention Institute at $\mathrm{BCH}$ to undergo a Pap test;

4. rigorous control of the cytologists performed by the cytopathology laboratory with a constant monitoring and training of professionals;

Cervical Cancer Screening Program in Brazil
5. systematic follow-up of the women participating in the prevention program using software specifically developed for that objective;

6. evaluation of the prevention and screening program organized through this software.

\section{Management of Abnormalities}

Cases with a repeated finding of cellular atypia in cytology are referred for colposcopic examination. Since 2014, we have implemented a few modifications, such as the addition to our protocol of HPV testing for certain conditions. For example, for ASCUS or LSIL, a repeat Pap test is performed, and if persistent we add highrisk (oncogenic) HPV testing. The women aged $\geq 30$ years with cytology in liquid medium with ASCUS and LSIL are automatically tested for high-risk HPV (hr-HPV; Cobas Test 4800, Roche). Positive cases are referred to colposcopy. Negative cases repeat the Pap test after 12 months.

Figures 5 and 6 depict the step-by-step process of the screening program and handling of the results of cytology tests. 


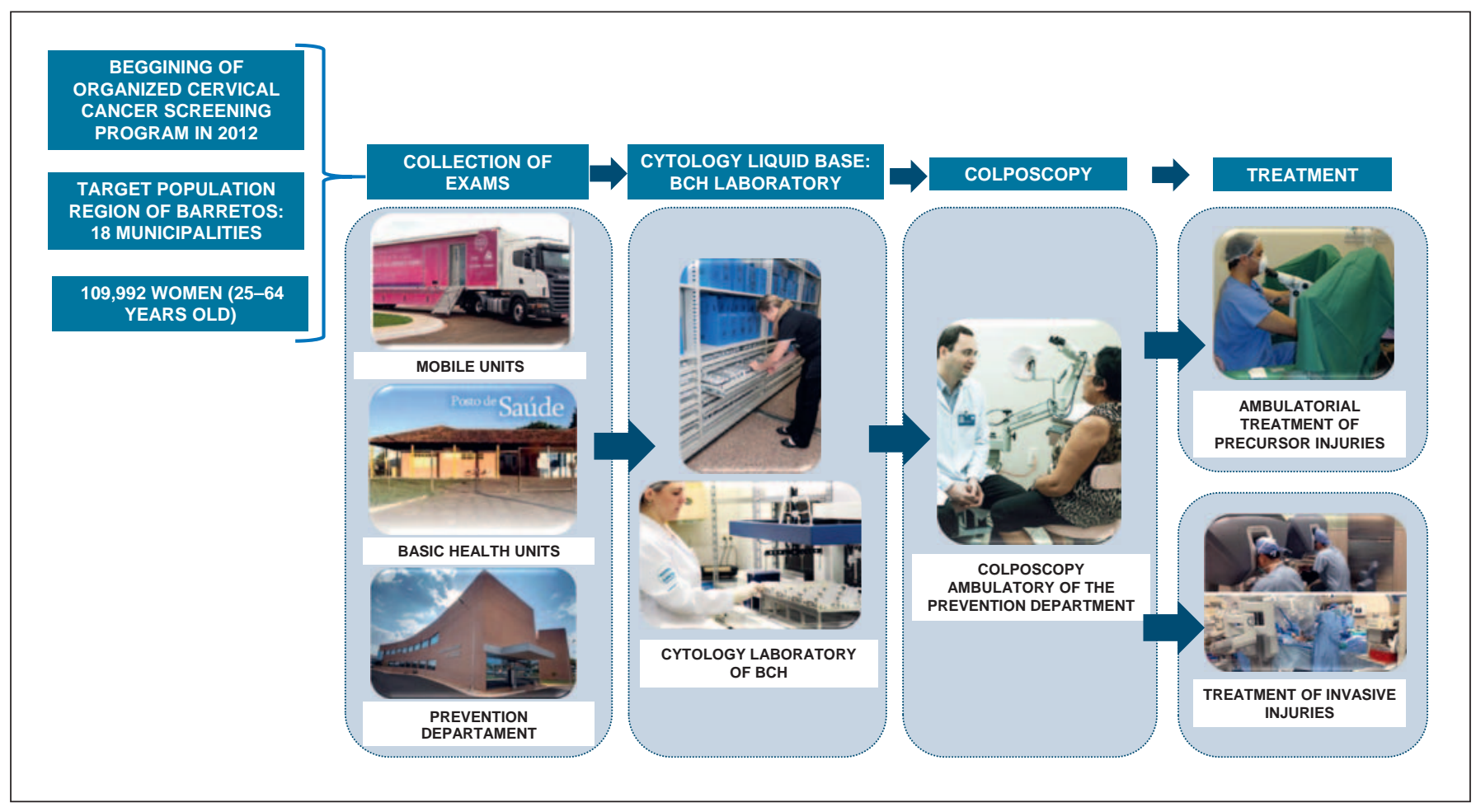

Fig. 5. Step-by-step depiction of the screening program for cervical cancer.

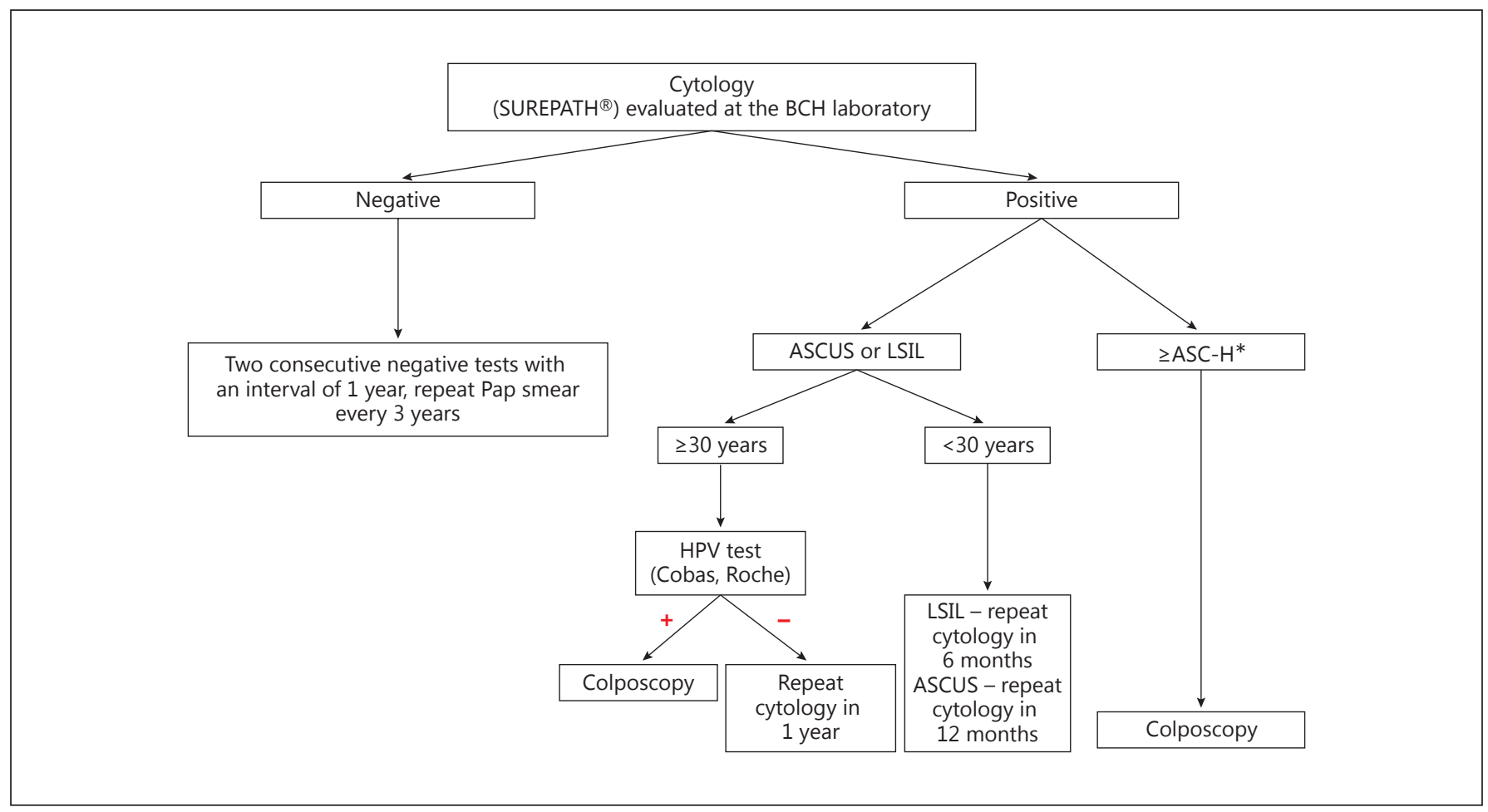

Fig. 6. Flowchart of the handling of the results of the cytology exams. ASC-H*, atypical squamous cells cannot exclude a high-grade squamous intraepithelial lesion. 


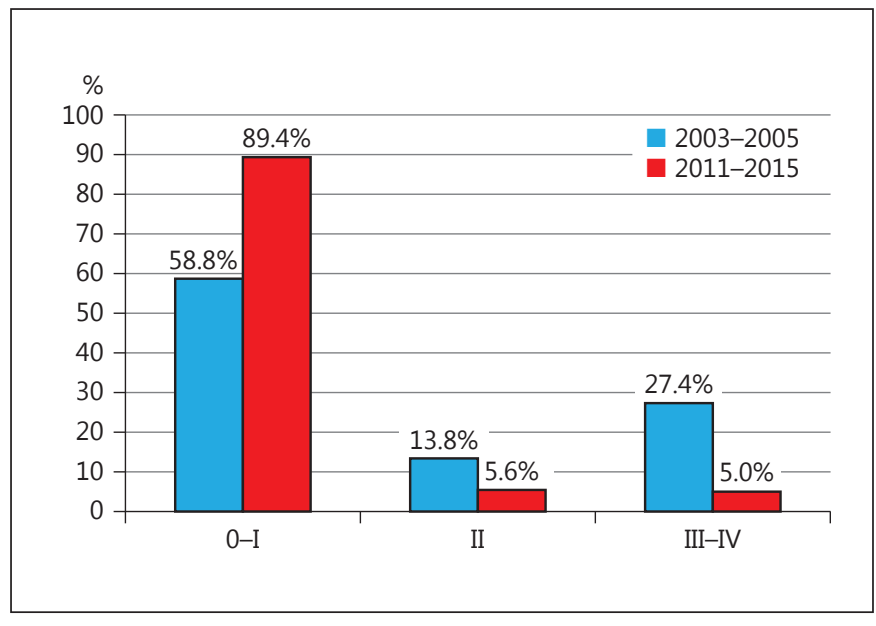

Fig. 7. Staging of diagnosed cases from 2003 to 2005, and from 2011 to 2015.

\section{Results}

Collectively, $\mathrm{BCH}$ has 4 units for cervical cancer prevention: Barretos, Campo Grande, Nova Andradina (Mato Grosso do Sul), and Porto Velho (Rondonia), and 8 mobile units to collect Pap test samples. All the units are coordinated by $\mathrm{BCH}$ using the model of organization previously depicted. All Pap test are collected in liquid medium and, in 2014, more than 160,000 Pap tests were analyzed and 2,900 colposcopy examinations were performed.

Between 2012 and 2015, the number of Pap tests performed in DRS-V were 33,636, 41,507, 41,713, and 40,024, respectively. Women $t$ not of target age (25-64 years) represent approximately $8.6 \%$ of the totals for each of these years. Specifically for DRS-V and the 18 municipalities in the Barretos region, the percentage of exams collected increased from 54.6 to $62.4 \%$ between 2012 and 2013, to $68.4 \%$ in 2014 , and $71 \%$ in 2015 . Per 1,000 Pap tests, 0.4 cases of invasive cancer are diagnosed $(2,444$ exams for 1 cancer case detected); for in situ carcinoma, 1.9 cases were identified (528 exams to detect 1 woman with a high-grade lesion). More importantly, between 2011 and $2015,89.4 \%$ of all carcinomas were detected at clinical stage 0 and I (in situ carcinoma), and only $5 \%$ at stages III and IV (Fig. 7).

Regarding the patients who were scheduled to repeat the Pap smear in 2015, out of 31,939 women, 29,921 repeated the exam (78\%), with $18 \%$ repeating the Pap within an adequate time, $41.1 \%$ after 30 days of the scheduled date, and $40.9 \%$ within 30 days of the scheduled date.

Cervical Cancer Screening Program in Brazil
The rates of unsatisfactory sample dropped from almost $0.83 \%$ using traditional smears to $0.14 \%$ of the total of samples collected with the introduction of liquid-based cytology. The index of positive cases increased from approximately $1.0 \%$ (below the international standards) to almost $4.0 \%$ (within international recommendations) $[12,13]$.

Once the organized system was implemented at $\mathrm{BCH}$, $98 \%$ of women attended their recall for colposcopy. In 2015,419 patients were referred for colposcopy and $97.6 \%$ of women attended the recall for colposcopy.

\section{Discussion}

There are many advantages related to the introduction of an organized program for cervical screening, and our results demonstrate many of them. An organized system depends on planning, the preparation of professionals, and implementation of rigorous controls for each step. This ranges from determining and identifying the target population, to determining how to reach this population, how to maintain the adherence of the population within the system, what kind of test to use, how to trace the population at risk and how to convoke those women detected with cervical abnormalities, and finally how, when and where to treat and follow up patients with cervical diseases [12].

In addition, Miller et al. [13] also highlight the importance of actions aimed at continuing the education of both the population and the professionals involved in all stages of the screening, or in the collection of tests, and the laboratory analysis of the tests. Therefore, $\mathrm{BCH}$ has continuously offered training courses for community agents, nurses, and physicians with content relating to the primary and secondary prevention of cervical cancer.

In 2012, BCH implemented an organized program to replace the opportunistic one that prevails throughout Brazil. Not surprisingly, the failures of Brazilian opportunistic cytology-based screening are under severe criticism due to the dismal performance of the laboratorial references, which do not contribute to a reduction in mortality [12].

The main limitation of this study is that the IBGE provides only the absolute numbers of women aged between 25 and 64 years $(109,992$ women) of the target population residing in the municipalities that are part of the organized screening. Thus, it is not possible to obtain the addresses of these women to check other pertinent information related to the epidemiological analyses. Therefore,

Acta Cytologica 2018;62:19-27 DOI: $10.1159 / 000480446$ 
we only worked with population coverage estimates and our population of women participating in the organized screening consists of those registered at our Prevention Institute.

The results herein discussed represent an obvious consequence of the work commenced in $\mathrm{BCH}$ many years ago (1994), using bikes, small busses, and (currently) large trucks (mobile units), and a serious commitment to quality in all steps of the screening process $[14,15]$. Despite the historical origin of the Prevention Institute, the results since 2012 show a constant baseline of a very high rate of in situ carcinoma (in situ clinical stage) among patients with cervical carcinomas that reflects, in part, the work focused in maintaining the commitment of subjects to the screening program [16].

The computer program developed to obtain the information needed to recruit women, as described previously, brings us many advantages. In addition to managing the screening program, it facilitates a routine procedure, reduces error, improves the quality of the services provided, and manages several types of information, which allows the creation of different databases enabling a constant evaluation of the prevention program.

Another point worth highlighting is the benefit of the introduction of liquid-based cytology. The preanalytical steps in the preparation of Pap test slides were tremendously optimized, substantially reducing the number inadequate preparations to less than $0.1 \%$. This creates a huge advantage considering that most of the women examined by $\mathrm{BCH}$ live in rural and remote areas, which makes a recall due to poor quality preparation a critical issue [17]. Additionally, to reduce to a minimum the possibility of cytological errors (not shown in this work), BCH implemented a computer-guided screening, which assists the cytotechnicians in the primary reading of the slides [18].

The positivity index was also raised from unacceptable indexes under $1.0 \%$ to international standards of almost $4.0 \%$, which represents a very satisfying result of the strategy to implement the best program recommended internationally [12]. Adherence is a critical issue because a lack of adherence can impact on the number of deaths from invasive cancer, and also significantly affect the efficacy of cancer prevention programs [12].

The implementation of HPV testing associated with the maintenance of educational initiatives for poor populations and the use of media resources (letter invitations, radio, television, etc.) can reduce the number of women remaining outside the programs $[19,20]$. The option of using liquid-based cytology also helps these initiatives to reduce disparities and facilitate the integration of many women into screening programs since certain HPV tests can be made inside the mobile units, either collected by a physician or nurse, or self-collected, which is a tremendous advance for zones without medical assistance [21].

Additionally, some self-sampling devices can be sent by regular mail to a woman's home and delivered back to the laboratories by the same means [22]. We introduced HPV screening for specific scenarios, as mentioned previously, and the results are very encouraging because the association of a positive hr-HPV test with the immunohistochemical markers p16/Ki-67 identified high-grade cervical intraepithelial neoplasia in women with a cervical cytology of ASCUS and LSIL, revealing 8.4\% with HSIL among ASCUS patients, and $22.1 \%$ with HSIL among the cytological LSIL group. Separately, we observed that both p16/ Ki-67 double staining and the hr-HPV DNA test had a similar performance in predicting high-grade cervical intraepithelial neoplasia among women with ASCUS [23, 24].

The persistent unfavorable indicators of incidence and mortality secondary to noncervical cancer indicate the need for an improvement and expansion of the current screening program. Although regional, the experience of the $\mathrm{BCH}$, which proposes a transition from opportunistic to organized testing, should be considered as a model for government initiatives in the rest of the country.

In addition, taking into account the heterogeneity and socioeconomic differences of the different regions of Brazil, the possibility of including other strategies within the tracing should be strongly evaluated. Numerous studies have shown the benefits of introducing HPV testing in population screening, especially among developing and resource-poor countries, which clearly have limitations in the quality of cytological tests.

Finally, the main restriction of our program for the prevention cervical cancer is still the lack of ideal coverage. We have not yet reached the target of $70 \%$ of women for this proposal, as recommended by international standards. These insufficient coverage rates are associated with a significant proportion of women being served by private health services in the Barretos region, which are not accounted for in the $\mathrm{BCH}$ files. On the other hand, there is still a contingent of women resistant to the performance of conventional cytology, which indicates that it is opportune to offer other screening modalities, such as self-collected HPV tests.

\section{Disclosure Statement}

The authors have no conflicts of interest to report in relation to this work.
26

Acta Cytologica 2018;62:19-27

DOI: $10.1159 / 000480446$
Possati-Resende et al. 


\section{References}

1 Ferlay J, Shin HR, Bray F, Forman D, Mathers C, Parkin DM: Estimates of worldwide burden of cancer in 2008: GLOBOCAN, 2008. Int J Cancer 2010;127:2893-2917.

2 Ferlay J, Soerjomataram I, Dikshit R, Eser S, Mathers C, Rebelo M, Bray F: Cancer incidence and mortality worldwide: sources, methods and major patterns in GLOBOCAN 2012. Int J Cancer 2015;136:E359-E386.

3 INCA: Estimativa 2016: Incidência de câncer no Brasil. Instituto Nacional de Câncer, 2016. http://www.inca.gov.br/estimativa/2016/.

4 INCA: Coordenação Geral de Ações Estratégicas. Coordenação de Prevenção e Vigilância. Instituto Nacional de Câncer, 2016. http://www2.inca.gov.br/wps/wcm/connect/ sobreinca/site/oinstituto/conselho_diretoria_executiva/direcao_geral/conprev.

5 Sankaranarayanan R, Black RJ, Parkin DM: Cancer Survival in Developing Countries. Lyon, IARC Press, 1988, p 15.

6 Hakama M, Miller AB, Day NE: Screening for Cancer of the Uterine Cervix. Lyon, IARC Press, 1986, p 76.

7 Instituto Brasileiro de Geografia e Estatística: Contas Regionais. Rio de Janeiro, IBGE, 2014.

8 Instituto Nacional de Câncer: Atlas de mortalidade por câncer. Rio de Janeiro, INCA, 2011. http://mortalidade.inca.gov.br/Mortalidade/.

9 World Health Organization: National Cancer Control Programmes: Policies and Managerial Guidelines, ed 2. Geneva, WHO, 2002.

10 Instituto Nacional de Câncer: Programa Nacional de Controle do Câncer do Colo do Útero. Rio de Janeiro, INCA 2011.

11 Macinko J, Starfied B, Shi L: The contribution of primary care systems to health outcomes within organization for economic cooperation and development (OECD) countries, 1970-1998. Health Serv Res 2003;38:831865.
12 Instituto Nacional de Câncer: Manual de gestão da qualidade para laboratório de citopatologia. Rio de Janeiro, INCA, 2012, p 188.

13 Miller AB, Nazeer S, Fonn S, Brandup-Lukanow A, Rehman R, Cronje H, Onsrud M: Report on consensus conference on cervical cancer screening and management. Int J Cancer 2000;86:440-447.

14 Branca M, Longatto-Filho A: Recommendations on quality control and quality assurance in cervical cytology. Acta Cytol 2015;59:361369.

15 Costa RFA, Longatto-Filho A, Pinheiro C, Zeferino LC, Fregnani JH: Historical analysis of the Brazilian cervical cancer screening program from 2006 to 2013: a time for reflection. PLoS One 2015; 10:e0138945.

16 Mauad EC, Nicolau SM, Gomes UA, da Costa Vieira RA, de Castro Mattos JS, Longatto-Filho A, Baracat EC: Can mobile units improve the strategies for cervical cancer prevention? Diagn Cytopathol 2010;38:727-730.

17 Mauad EC, Nicolau SM, Moreira LF, Haikel RL Jr, Longatto-Filho A, Baracat EC: Adherence to cervical and breast cancer programs is crucial to improving screening performance. Rural Remote Health 2009;9:1241.

18 Fregnani JH, Scapulatempo C, Haikel RL Jr, Saccheto T, Campacci N, Mauad EC, Longatto-Filho A; RODEO Study Team: Could alarmingly high rates of negative diagnoses in remote rural areas be minimized with liquidbased cytology? Preliminary results from the RODEO Study Team. Acta Cytol 2013;57:69_ 74 .
19 Stein MD, Fregnani JH, Scapulatempo C, Mafra A, Campacci N, Longatto-Filho A; RODEO Study Team from Barretos Cancer Hospital: Performance and reproducibility of gynecologic cytology interpretation using the FocalPoint system: results of the RODEO Study Team. Am J Clin Pathol 2013;140:567571.

20 Alfaro KM, Gage JC, Rosenbaum AJ, Ditzian LR, Maza M, Scarinci IC, Miranda E, Villalta S, Felix JC, Castle PE, Cremer ML: Factors affecting attendance to cervical cancer screening among women in the Paracentral Region of El Salvador: a nested study within the CAPE HPV screening program. BMC Public Health 2015;15:1058.

21 López-Torres HJ, Sánchez OMP, Rabanales SJ, Simarro HMJ, López-Torres LJ, Campos RM: Effectiveness of three interventions in improving adherence to cervical cancer screening. Eur J Cancer Prev 2016;25:423429.

22 Lorenzi AT, Fregnani JH, Possati-Resende JC, Neto CS, Villa LL, Longatto-Filho A: Self-collection for high-risk HPV detection in Brazilian women using the careHPV ${ }^{\mathrm{TM}}$ test. Gynecol Oncol 2013;131:131-134.

23 Giorgi RP, Fortunato C, Barbarino P, Boveri S, Caroli S, Del Mistro A, Ferro A, Giammaria C, Manfredi M, Moretto T, Pasquini A, Sideri M, Tufi MC, Cogo C, Altobelli E; HPV Selfsampling Italian Working Group: Self-sampling to increase participation in cervical cancer screening: an RCT comparing home mailing, distribution in pharmacies, and recall letter. Br J Cancer 2015;112:667-675.

24 Possati-Resende JC, Fregnani JH, Kerr LM, Mauad EC, Longatto-Filho A, ScapulatempoNeto C: The accuracy of p16/Ki-67 and HPV test in the detection of CIN2/3 in women diagnosed with ASC-US or LSIL. PLoS One 2015;10:e134445.
Cervical Cancer Screening Program in Brazil
Acta Cytologica 2018;62:19-27

DOI: $10.1159 / 000480446$ 